Macdonald, M. and McKay, R.J. and Vasile, M. and Bosquillon de Frescheville, Francois (2010) Extension of the sun-synchronous Orbit. Journal of Guidance, Control and Dynamics . ISSN 0731-5090

http://strathprints.strath.ac.uk/26495/

Strathprints is designed to allow users to access the research output of the University of Strathclyde. Copyright (C) and Moral Rights for the papers on this site are retained by the individual authors and/or other copyright owners. You may not engage in further distribution of the material for any profitmaking activities or any commercial gain. You may freely distribute both the url (http://strathprints.strath.ac.uk) and the content of this paper for research or study, educational, or not-for-profit purposes without prior permission or charge. You may freely distribute the url (http://strathprints.strath.ac.uk) of the Strathprints website.

Any correspondence concerning this service should be sent to The Strathprints Administrator: eprints@cis.strath.ac.uk 


\title{
Extension of the Sun-Synchronous Orbit
}

\author{
Malcolm Macdonald ${ }^{1}$, Robert $\mathrm{M}^{\mathrm{c}} \mathrm{Kay}^{2}$ \\ University of Strathclyde, Glasgow, G1 1XJ, Scotland, E.U. \\ Massimiliano Vasile ${ }^{3}$ \\ University of Glasgow, Glasgow, G12 8QQ, Scotland, E. U. \\ François Bosquillon de Frescheville ${ }^{4}$ \\ European Space Agency, 64293 Darmstadt, Germany, E. U.
}

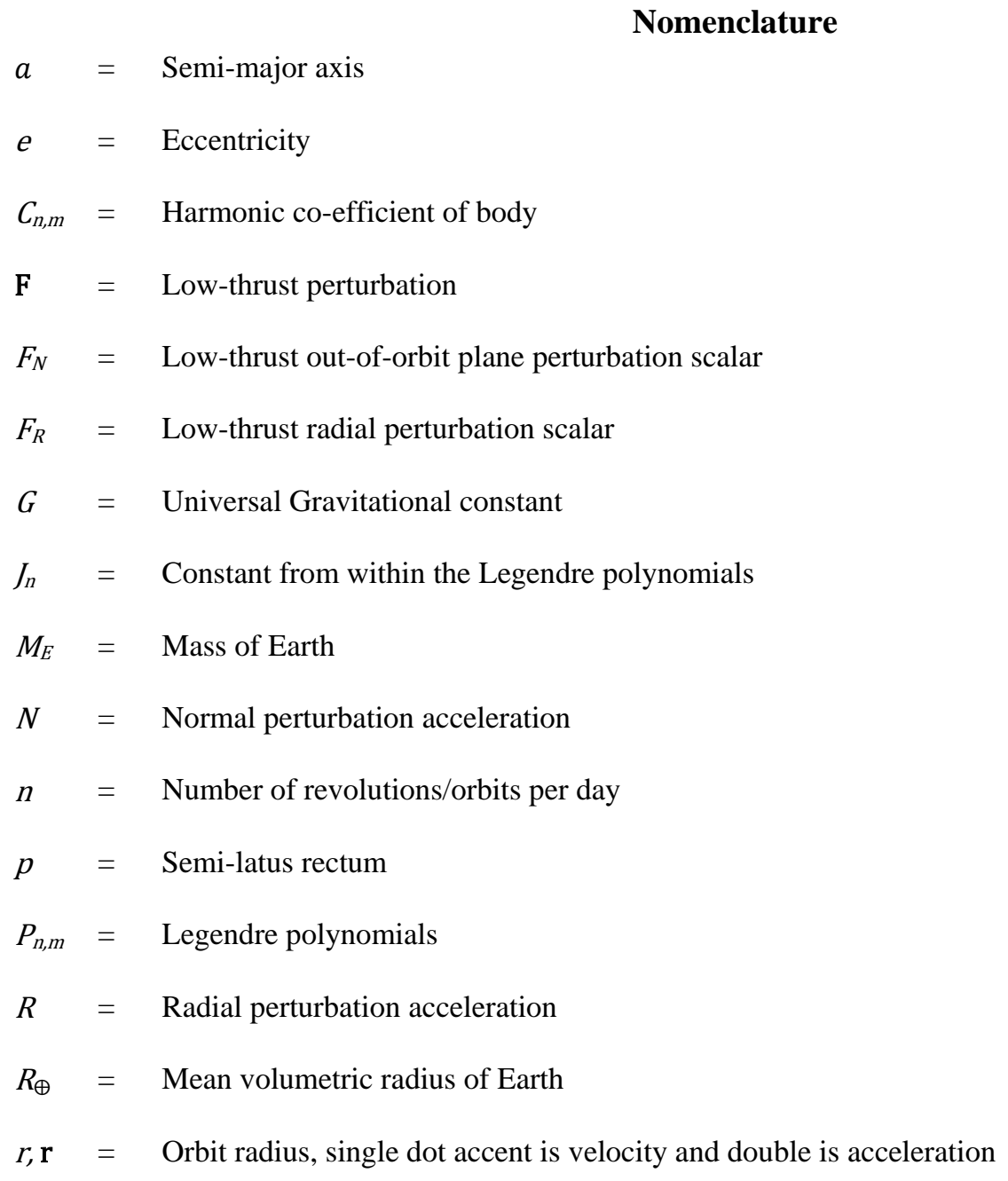

\footnotetext{
${ }^{1}$ Associate Director, Advanced Space Concepts Laboratory, Department of Mechanical Engineering. malcolm.macdonald.102@strath.ac.uk, Senior Member AIAA.

${ }^{2}$ Research Fellow, Advanced Space Concepts Laboratory, Department of Mechanical Engineering.

Robert.j.mckay@strath.ac.uk.

${ }^{3}$ Senior Lecturer, Space Advanced Research Team, Department of Aerospace Engineering. m.vasile@ aero.gla.ac.uk

${ }^{4}$ Future Studies Operations Concept Engineer, European Space Operations Centre, Human Spaceflight and

Exploration Department, Robert-Bosch-Str. 5, Francois.Bosquillon.de.Frescheville@esa.int.
} 


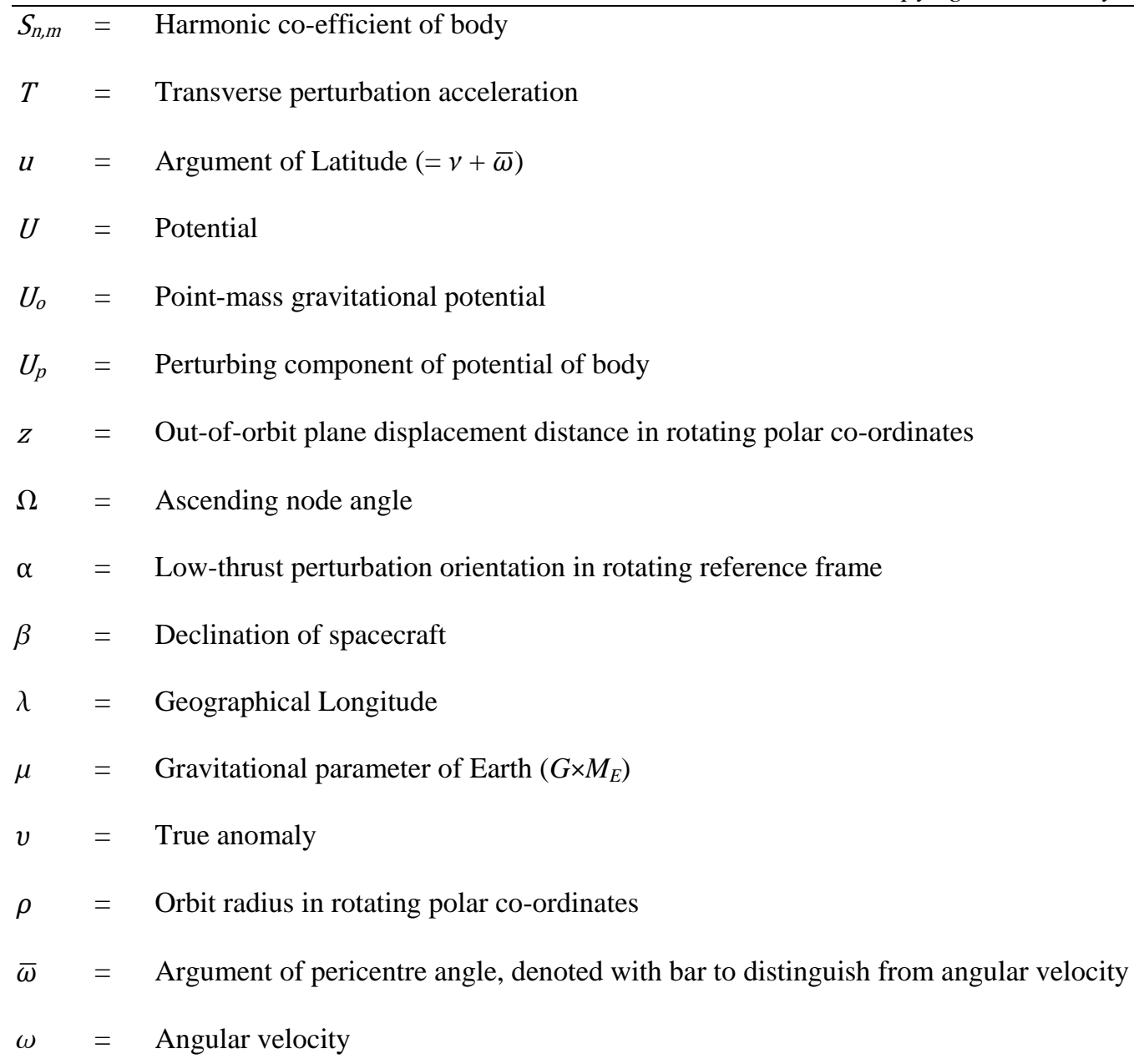

All units are SI.

\section{Introduction}

$\mathrm{T}^{\mathrm{H}}$

HROUGH careful consideration of the orbit perturbation force due to the oblate nature of the primary body a secular variation of the ascending node angle of a near-polar orbit can be induced without expulsion of propellant. Resultantly, the orbit perturbations can be used to maintain the orbit plane in, for example, a near-perpendicular (or at any other angle) alignment to the Sun-line throughout the full year of the primary body; such orbits are normally termed Sun-synchronous orbits [1,2]. Sun-synchronous orbits about the Earth are typically near-circular Low-Earth Orbits (LEOs), with an altitude of less than $1500 \mathrm{~km}$. It is normal to design a LEO such that the orbit period is synchronised with the rotation of the Earth's surface over a given period, such that a repeating ground-track is established. A repeating ground-track, together with the near-constant illumination conditions of the ground-track when observed from a Sun-synchronous orbit, enables repeat observations of a target over an extended period under 
Extension of the Sun-Synchronous Orbit author pre-print to Journal Guidance Control and Dynamics Copyright $\odot 2010$ by Malcolm Macdonald.

similar illumination conditions [1,2]. For this reason, Sun-synchronous orbits are extensively used by Earth Observation (EO) platforms, including currently the Environmental Satellite (ENVISAT), the second European Remote Sensing satellite (ERS-2) and many more.

By definition, a given Sun-synchronous orbit is a finite resource similar to a geostationary orbit. A typical characterising parameter of a Sun-synchronous orbit is the Mean Local Solar Time (MLST) at descending node, with a value of 1030 hours typical. Note that ERS-1 and ERS-2 used a MLST at descending node of 1030 hours \pm 5 minutes, while ENVISAT uses a 1000 hours \pm 5 minutes MLST at descending node [3]. Following selection of the MLST at descending node and for a given desired repeat ground-track, the orbit period and hence the semi-major axis are fixed, thereafter assuming a circular orbit is desired it is found that only a single orbit inclination will enable a Sun-synchronous orbit [2]. As such, only a few spacecraft can populate a given repeat ground-track Sunsynchronous orbit without compromise, for example on the MLST at descending node. Indeed a notable feature of on-going studies by the ENVISAT Post launch Support Office is the desire to ensure sufficient propellant remains at end-of-mission for re-orbiting to a graveyard orbit to ensure the orbital slot is available for future missions [4].

An extension to the Sun-synchronous orbit is considered using an undefined, non-orientation constrained, lowthrust propulsion system. Initially the low-thrust propulsion system will be considered for the free selection of orbit inclination and altitude while maintaining the Sun-synchronous condition. Subsequently the maintenance of a given Sun-synchronous repeat-ground track will be considered, using the low-thrust propulsion system to enable the free selection of orbit altitude. An analytical expression will be developed to describe these extensions prior to then validating the analytical expressions within a numerical simulation of a spacecraft orbit. Finally, an analysis will be presented on transfer and injection trajectories to these orbits.

\section{Satellite Motion about an Oblate Body}

The gravitational potential may be written as [1]

$$
U(r, \beta, \lambda)=\frac{\mu}{r} \sum_{n=0}^{\infty} \sum_{m=0}^{\infty}\left(\frac{R_{E}}{r}\right)^{n}\left(C_{n, m} \cos m \lambda+S_{n, m} \sin m \lambda\right) P_{n, m} \sin \beta
$$

which, for a body possessing axial symmetry may be written, for a point external to it, as

$$
U(r, \beta)=\frac{\mu}{r}\left[1-\sum_{n=0}^{\infty} J_{n}\left(\frac{R_{E}}{r}\right)^{n} P_{n} \sin \beta\right]
$$

This assumption is valid for Earth as the influence of periodic effects (tesseral and sectorial harmonics) can be neglected for most orbits, with the notable exception of geostationary orbits. It is thereafter found that 


$$
\begin{aligned}
U(r, \beta)=\frac{\mu}{r}[1 & -J_{2} \frac{1}{2}\left(\frac{R_{\oplus}}{r}\right)^{2}\left(3 \sin ^{2} \beta-1\right) \\
& -J_{3} \frac{1}{2}\left(\frac{R_{\oplus}}{r}\right)^{3}\left(5 \sin ^{3} \beta-3 \sin \beta\right) \\
& -J_{4} \frac{1}{8}\left(\frac{R_{\oplus}}{r}\right)^{4}\left(3-30 \sin ^{2} \beta+35 \sin ^{2} \beta\right)-\ldots
\end{aligned}
$$

Using spherical triangle laws and considering only the first order terms, Eq. 3 reduces to

$$
U(r, \beta)=U_{o}+U_{p}=\frac{\mu}{r}-J_{2} \frac{\mu R_{\oplus}^{2}}{2 r^{3}}\left(3 \sin ^{2} i \sin ^{2} u-1\right)
$$

A Sun-synchronous orbit requires that the rate of change of the ascending node match the mean rate of rotation of the Sun within an Earth-centred inertial reference frame; $(2 \pi / 365.25)$. The ascending node angle can be described within an Earth-centred inertial reference frame through the Gaussian form of the variational equations of the classic orbital elements. Selecting the positioning-fixing element to be true anomaly the rate of change of the ascending node angle is [5]

$$
\frac{d \Omega}{d v}=\frac{r^{3}}{\mu p \sin i} \sin (v+\bar{\omega}) N
$$

Thus, $U_{p}$ within Eq. 4 is required in terms of the spacecraft centred $R T N$ co-ordinate system and is obtained by differentiation of the potential with respect to the spacecraft centred $R T N$ co-ordinate system. The disturbing force components due to $J_{2}$ are thus

$$
\begin{aligned}
& R_{J_{2}}=\frac{3}{2} J_{2} \frac{\mu R_{\oplus}^{2}}{r^{4}}\left(3 \sin ^{2} i \sin ^{2} u-1\right) \\
& T_{J_{2}}=-\frac{3}{2} J_{2} \frac{\mu R_{\oplus}^{2}}{r^{4}} \sin ^{2} i \sin ^{2} u \\
& N_{J_{2}}=-\frac{3}{2} J_{2} \frac{\mu R_{\oplus}^{2}}{r^{4}} \sin 2 i \sin u
\end{aligned}
$$

Through a combination of Eq. 5 and Eq. 8, and assuming that the change in other orbit elements is small over the integral, the standard Sun-synchronous orbit can be found as

$$
i=\cos ^{-1}\left[-\frac{2}{3} \frac{\Delta \Omega}{J_{2}} \frac{a^{7 / 2}\left(1-e^{2}\right)^{2}}{R_{\oplus}^{2} \sqrt{\mu}}\right]
$$

where, $\Delta \Omega$ is the mean rotation rate of the Sun within an Earth-centred inertial reference frame per second. 
Extension of the Sun-Synchronous Orbit author pre-print to Journal Guidance Control and Dynamics Copyright $\odot 2010$ by Malcolm Macdonald.

\section{Low-Thrust Rotation of Ascending Node Angle with $\mathbf{J}_{\mathbf{2}}$}

From Eq. 5, and noting that $\sin i \geq 0$ for $0 \leq i<\pi$, a secular variation of the ascending node angle over the orbit period is obtained when the low-thrust propulsion system provides an out-of-plane perturbation which switches sign as a function of $\sin (v+\bar{\omega})[6]$. The combined $J_{2}$ and low-thrust out-of-plane perturbation is thus

$$
N=-\frac{3}{2} J_{2} \frac{\mu R_{\oplus}^{2}}{r^{4}} \sin 2 i \sin u+F_{N} \operatorname{sgn}[\sin (v+\bar{\omega})]
$$

Thereafter, noting that

$$
r=\frac{p}{1+e \cos v}
$$

the variation of ascending node angle over the orbit period can be found to be

$$
\begin{aligned}
(\Delta \Omega)_{0}^{2 \pi}= & \frac{p^{2}}{\mu \sin i} F_{N} \int_{0}^{2 \pi} \frac{\sin (v+\bar{\omega})}{(1+e \cos v)^{3}} \operatorname{sgn}[\sin (v+\bar{\omega})], d v \\
& -3 J_{2}\left(\frac{R_{\oplus}}{p}\right)^{2} \cos i \int_{0}^{2 \pi} \sin ^{2}(v+\bar{\omega})(1+e \cos v), d v
\end{aligned}
$$

with the second integral on the right-hand side of Eq. 12 equalling $\pi$. To solve the first integral, independent of the physical meaning of the variables, it is assumed that $0 \leq e<1$. Thereafter Eq. 12 becomes

$$
(\Delta \Omega)_{0}^{2 \pi}=\frac{4 p^{2}}{\mu\left(-1+e^{2}\right)^{2}} \frac{\cos \bar{\omega}}{\sin i} F_{N}-3 \pi J_{2}\left(\frac{R_{\oplus}}{p}\right)^{2} \cos i
$$

Switching the rate of change of ascending node angle per rotation to per second and re-arranging, Eq. 13 then becomes

$$
\Delta \Omega=\frac{1}{2 \pi}\left[4 \frac{\cos \bar{\omega}}{\sin i} \sqrt{\frac{\mathrm{a}}{\mu}} F_{N}-\frac{3 \pi J_{2} \sqrt{\mu} \cos \mathrm{i}}{\sqrt{a^{3}}}\left(\frac{R_{\oplus}}{\mathrm{p}}\right)^{2}\right]
$$

Eq. 14 can be solved analytically for semi-major axis. However, as the function has eight roots such a derivation would be convoluted and it is solved numerically herein, allowing determination of the enabled Sun-synchronous orbits over a range of semi-latus rectum and inclination for a given, continuous low-thrust perturbation following the switching control law detailed above.

Assuming a circular orbit, Eq. 14 is solved numerically within Figure 1. From Figure 1, it is seen that for a given altitude the addition of a continuous low-thrust perturbation enables the use of different orbit inclinations. For example, at $n=15$ the natural Sun-synchronous orbit has altitude approximately $561 \mathrm{~km}$, assuming mean volumetric radius of Earth, and inclination 97.6 degrees. However, by application of a continuous low-thrust perturbation of magnitude $0.34 \mathrm{~mm} \mathrm{~s}^{-2}$ the Sun-synchronous orbit can be shifted to $n=14$, altitude $888 \mathrm{~km}$ while maintaining the same orbit inclination. 


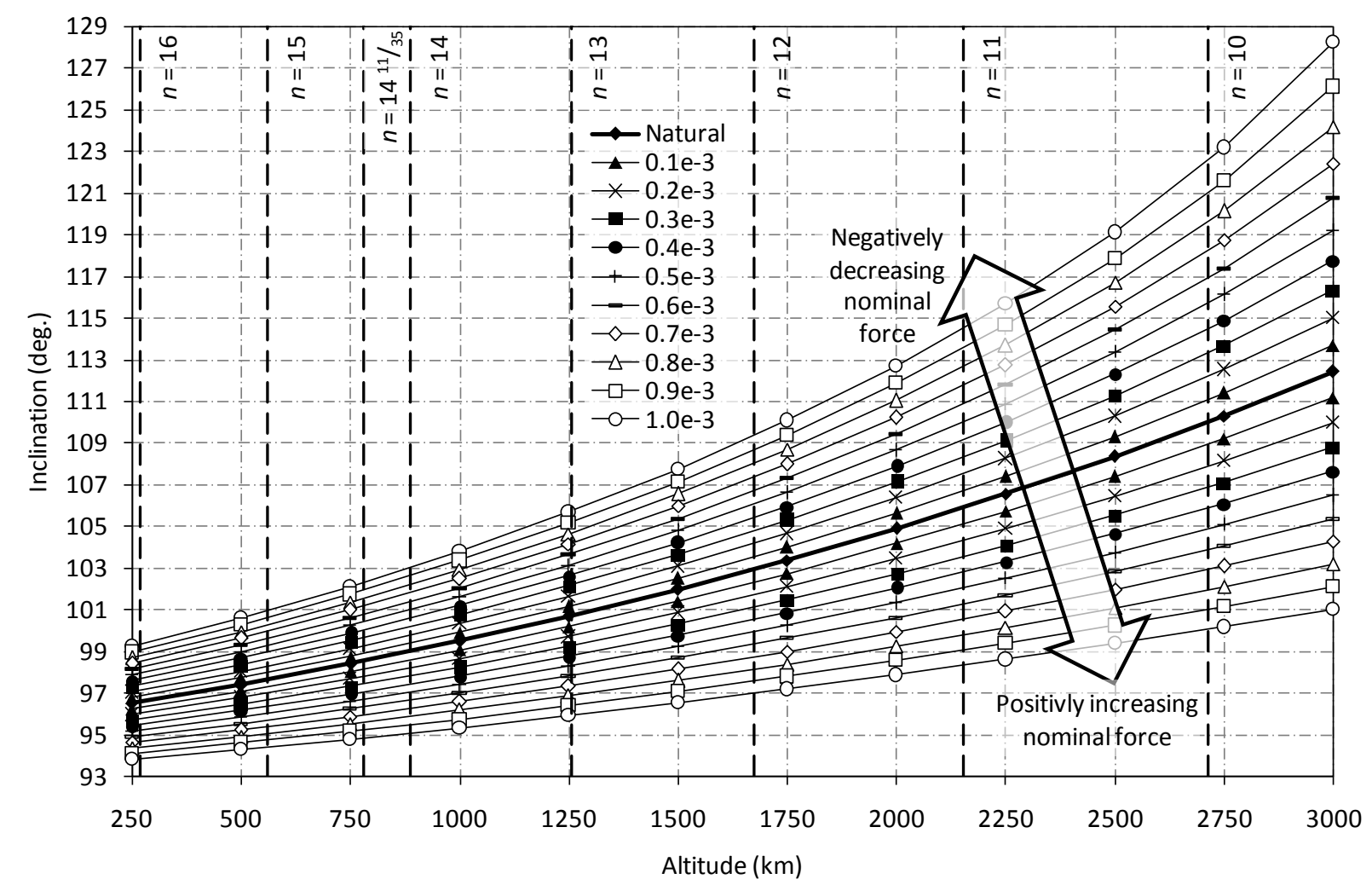

Figure 1 Sun-synchronous circular orbits with continuous acceleration over a range of values from $-1 \mathrm{~mm} \mathrm{~s}^{-2}$ to $1 \mathrm{~mm} \mathrm{~s}^{-2}$, contours of number of revolutions per day $(n)$ also shown

\section{Displaced Sun-Synchronous Orbits}

The use of continuous low-thrust has been shown to enable extension of Sun-synchronous orbits to alternative inclinations for a given repeat cycle. However, a continuous low-thrust propulsion system can be used to further displace the orbit from those derived using Eq. 14, enabling the orbit altitude to be varied for a given repeat-ground track through consideration of displaced two-body orbits. A displaced two-body orbit is an orbit which does not satisfy the conditions of a Keplerian orbit, instead using a low-thrust propulsion system to counteract the effects of gravity and to, for example, orbit at a period consistent with a different orbit semi-major axis. Thus, Sunsynchronous orbits (natural or extended) can be displaced such that the orbit altitude is changed without impacting the orbit period, hence maintaining a desired repeat cycle but at an inclination and altitude of choice. A displaced orbit, in the two-body problem, with an orbit period fixed by the reference orbit is termed a Type-III displaced orbit $[7,8]$.

The equation of motion of a satellite in the rotating reference frame, as shown in Figure 2, may be written as 
$\ddot{\mathbf{r}}+2 \boldsymbol{\omega} \times \dot{\mathbf{r}}=-\nabla U+\mathbf{F}$

It is noted that within Figure 2 the out-of-plane displacement is assumed to be from an Equatorial orbit, i.e. inclination zero. However, as the inclination does not alter the analysis it can be considered a free parameter, thus allowing inclination to be considered separately, as per a Sun-Synchronous orbit. From Ref. $7 \&$, and assuming a circular orbit, Eq. 15 reduces to

$$
\nabla U=\mathbf{F}
$$

where

$$
U(\rho, z ; \omega)=-\left(\frac{1}{2}(\omega \rho)^{2}+\frac{\mu}{r}\right)
$$

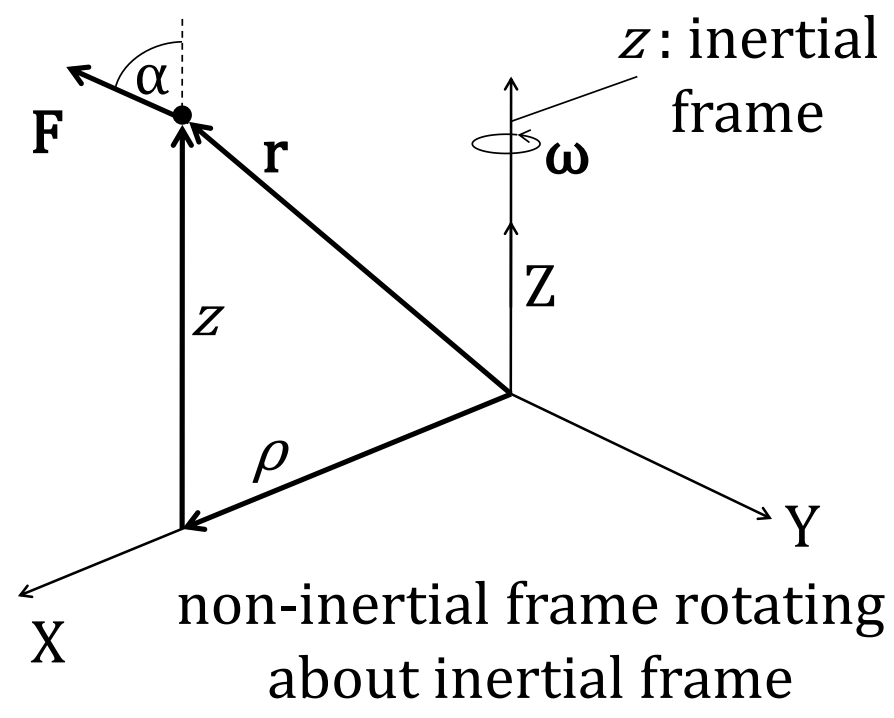

Figure 2 Rotating polar co-ordinate system

For a Type-III equilibrium, where $\rho$ and $z$ are the free parameters

$$
|\mathbf{F}|=\sqrt[2]{\rho^{2}\left(\omega_{0}-\omega_{*}\right)^{2}+z^{2} \omega_{*}^{4}}
$$

with the perturbation direction given by

$$
\tan \alpha=\frac{\rho}{z}\left[1-\left(\frac{\omega_{0}}{\omega_{*}}\right)^{2}\right]
$$

where, $\omega_{*}=\sqrt[2]{\left(\mu / \rho^{3}\right)}, \omega_{0}=\sqrt[2]{\left(\mu / r^{3}\right)}$ and $r$ is the reference orbit radius against which the displaced orbit shall be synchronous. 
Extension of the Sun-Synchronous Orbit author pre-print to Journal Guidance Control and Dynamics Copyright $\odot 2010$ by Malcolm Macdonald.

To maintain a given repeat ground-track, the vertical, or out-of-plane displacement from the reference orbit is zero $(z=0)$ while the desired thrust is along the radial direction. Recall that the required thrust for a non-natural Sun-synchronous orbit is out of the orbit plane and hence decoupled from the thrust required for a displaced orbit. From Eq. 14 and Figure 1 for an out-of-plane perturbation of magnitude $0.2 \mathrm{~mm} \mathrm{~s}^{-2}$ at $n=14$ a Sun-synchronous orbit can be achieved at orbit altitude $888 \mathrm{~km}$ and inclination 98.2 degrees or 99.8 degrees, i.e. \pm 0.79 degrees, depending on the orientation of the low-thrust propulsion. Using Eq. 18 it is found that for a further orbit perturbation of magnitude $0.2 \mathrm{~mm} \mathrm{~s}^{-2}$, in the radial direction, the orbit altitude can be varied by up to $64 \mathrm{~m}$ without altering the orbit period or ground-track. Similarly, an altitude variation of $1 \mathrm{~km}$ can be achieved by a radial orbit perturbation of magnitude $3.1 \mathrm{~mm} \mathrm{~s}^{-2}$. Clearly large variations in the orbit altitude require prohibitively large acceleration magnitudes, however small variations could be used for EO formation flying applications, such as distributed synthetic aperture radar. Finally, it is noted that each of the above steps allows the spacecraft to move from simply occupying a length of space around the Earth, i.e. a standard orbit, to initially an area of space defined as a segment of the surface of a sphere, i.e. variable inclination. To secondly, a volume of space defined as a segment of a thin-walled sphere, i.e. variable inclination and altitude whilst always maintain a constant groundtrack. Thus, the tracking of such an agile, unknown Earth observation (i.e. hostile military reconnaissance) spacecraft would be significantly more complex.

\section{Numerical Simulation}

The analytical analysis was examined within a numerical simulation for validity. The numerical simulation propagates the spacecraft position using an explicit, variable step-size Runge-Kutta $(4,5)$ formula, the DormandPrice pair (a single step method) [9], to integrate the modified equinoctial equations of motion in the Gauss' form $[10,11]$. The spacecraft trajectory was propagated with consideration to Lunar and Solar gravity as point masses and the Earth's oblateness up to the $18^{\text {th }}$ order (zonal and tesseral). No other perturbations are considered; however, the position of the Sun was corrected for the eccentricity of Earth's orbit.

The ENVISAT spacecraft has a nominal ground-track repeat period of 35 days $\left(n=14_{35}^{11}\right)$, giving a nominal altitude (assuming mean volumetric radius of Earth and a circular orbit) of $781 \mathrm{~km}$ and, for a natural SunSynchronous orbit, an inclination of 98.5 degrees. From Eq. 14 and Figure 1, if a continuous low-thrust perturbation of magnitude $0.2 \mathrm{~mm} \mathrm{~s}^{-2}$ is considered, it is possible to fix the orbit inclination and vary the altitude by plus 190.2 $\mathrm{km}$ to minus $165.9 \mathrm{~km}$. However, such a variation would alter the repeat ground-track period. Therefore, fixing the 
Extension of the Sun-Synchronous Orbit author pre-print to Journal Guidance Control and Dynamics Copyright $\odot 2010$ by Malcolm Macdonald.

orbit altitude the inclination can be varied by \pm 0.74 degrees. Figure 3 shows the separation angle between the ascending node of a natural, uncontrolled Sun-synchronous orbit similar to that of ENVISAT and the desired value, along with the similar separation angle for an uncontrolled Sun-Synchronous orbit with the same repeat groundtrack period at inclination 97.8 degrees, enabled by an acceleration magnitude of $0.2 \mathrm{~mm} \mathrm{~s}^{-2}$. It is seen that both scenarios presented in Figure 3 result in the ascending node angle rotating faster than desired. However, it is of note that the divergence of the non-natural orbit from the desired state is non-linear, as opposed to the natural orbit. It is of further note that the inclination, not shown, exhibits a long-period oscillation in the natural Sun-synchronous orbit due to $3^{\text {rd }}$ body gravity effects. However, in the forced scenario, while the inclination maintains a long-period oscillation the trend is a near-linear incremental drift of just over +0.1 degrees in the year.

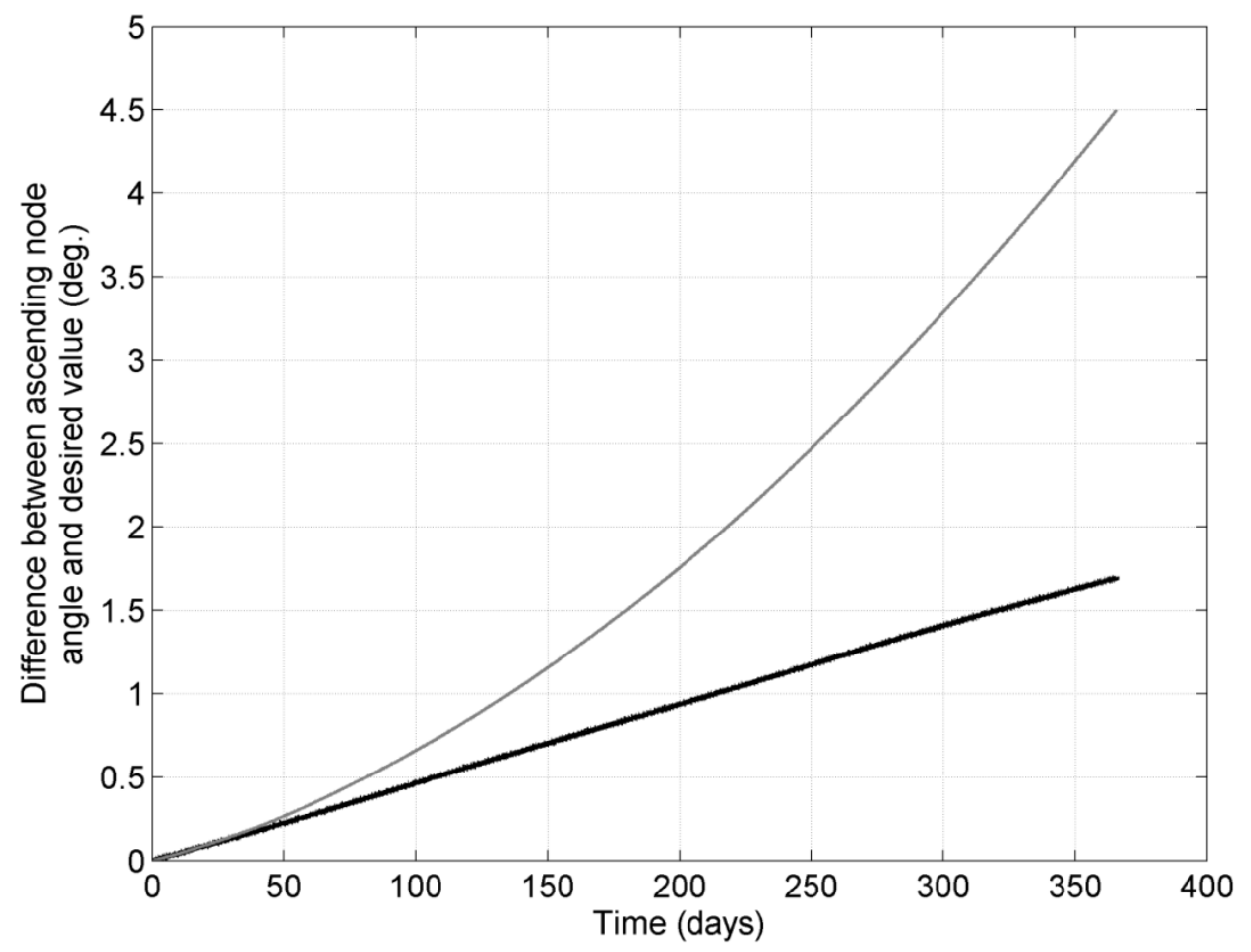

Figure 3 Separation angle between ascending node and desired value, black line is a natural, uncontrolled, Sun-Synchronous orbit, grey line is non-natural, uncontrolled, Sun-Synchronous orbit

An orbit displaced $+50 \mathrm{~m}$ in the radial direction from the above non-natural Sun-synchronous orbit but with the same orbit period is now considered, i.e. a Type-III displaced orbit. The initial conditions of such a numerical simulation must be carefully considered, as the orbital velocity must be set to conserve the angular velocity of the reference orbit. From Eq. 18, an orbit perturbation of magnitude $0.16 \mathrm{~mm} \mathrm{~s}^{-2}$ is required in the negative radial 
Extension of the Sun-Synchronous Orbit author pre-print to Journal Guidance Control and Dynamics Copyright $(2) 2010$ by Malcolm Macdonald.

direction, in addition an orbit perturbation of magnitude $(0.2+5.101 \mathrm{e}-5) \mathrm{mm} \mathrm{s}^{-2}$ out of the orbit plane is required to enable the Sun-synchronous rotation; the additional term in the out-of-plane perturbations accounts for the additional altitude of the displaced spacecraft. Figure 4 shows the uncontrolled separation distance between a spacecraft on the displaced orbit and a reference spacecraft on the non-natural Sun-synchronous orbit over a twelve hour period. Figure 4 shows that the inter-spacecraft distance drops below $45 \mathrm{~m}$ on the first orbit, with the interspacecraft distance oscillation magnitude increasing over subsequent orbits. The ascending node angle of the displaced spacecraft after 60 days was found to have drifted less than one arcminute behind the non-natural Sunsynchronous orbit. It is of note that if the orbit perturbations are removed and only the above radial perturbation applied to the displaced spacecraft the inter-spacecraft distance varies within the bounds of the numerical calculation error, therefore clearly suggesting that a simple control system should be able to maintain the nominal interspacecraft distance through the use of further low-thrust. Finally, the scenario presented in Figure 4 was repeated for a spacecraft displaced from the ENVISAT orbit, requiring only the radial thrust component to maintain the orbit displacement, and found to replicate Figure 4 to a very high-level of precision.

\section{A. Orbit Insertion Analysis}

The transfer to the displaced orbit has been split into two manoeuvres: an asynchronous transfer from an inclination of 98.5 degrees to an inclination of 97.8 degrees and a synchronous transfer from the orbit at 97.8 degrees of inclination to an orbit with equal inclination but an altitude increased of $50 \mathrm{~m}$. The low-thrust transfer was performed with a direct transcription method based on Finite Elements in Time generated on spectral basis [12, 13]. The equinoctial equations of motion in the Gauss' form were used to describe the spacecraft motion [10, 11]. The specific impulse of the engine was set to $3000 \mathrm{~s}$ and the maximum thrust level to $0.258 \mathrm{~N}$ on an initially 1000 $\mathrm{kg}$ spacecraft.

The asynchronous inclination change means that the spacecraft reaches the new inclination with a small phase difference with respect to the departure orbit. The inclination change takes 257 hours and requires $3.8 \mathrm{~kg}$ of propellant. The altitude change is synchronous and is performed over one full revolution, about 1.67 hours, and requires 53 grams of propellant. 


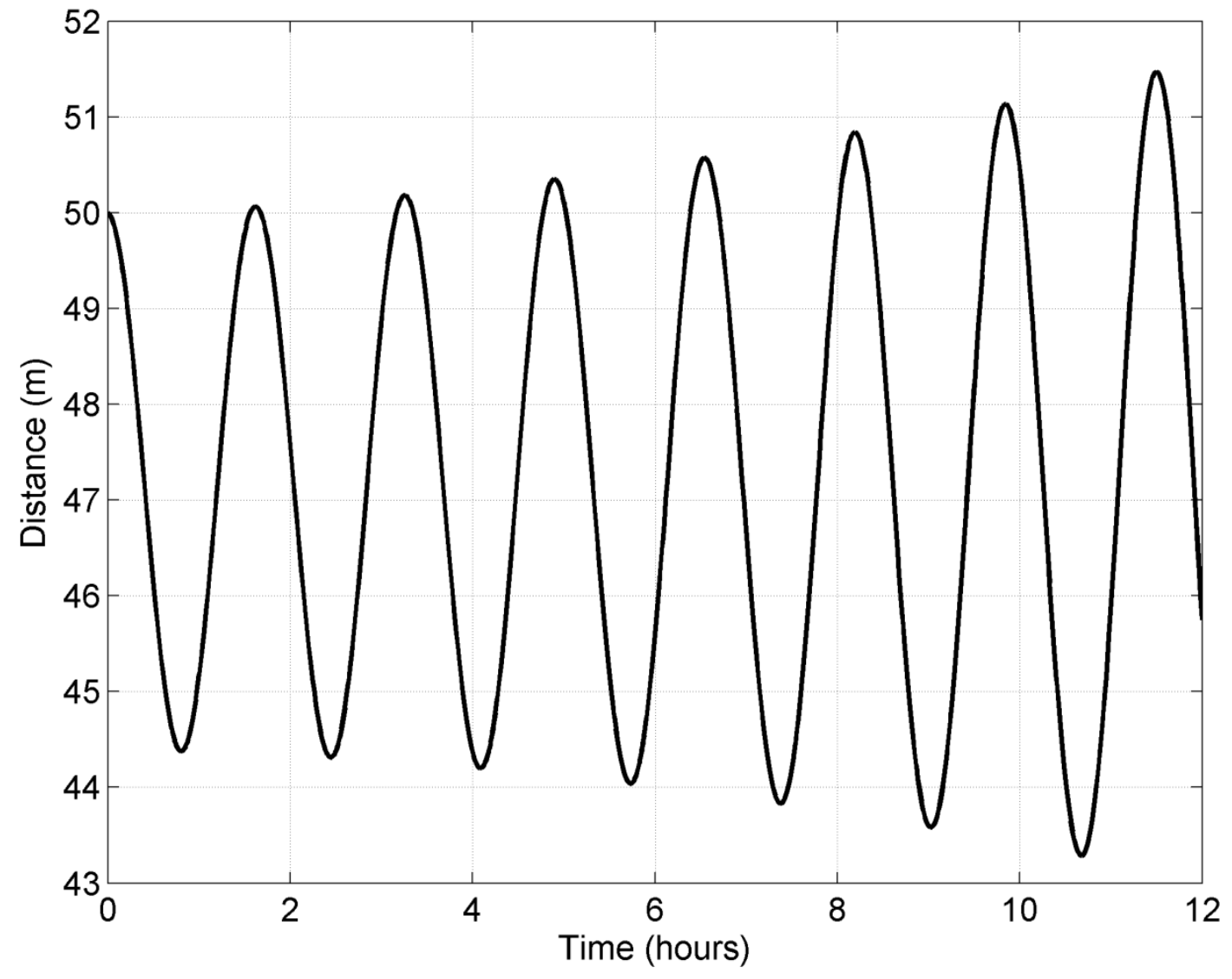

Figure 4 Inter-spacecraft distance between an ENVISAT-like non-natural Sun-synchronous orbit and a second spacecraft on a Type-III, 50 m displaced orbit

\section{Discussion}

Recently, the Committee on Earth Observation Satellites (CEOS) and the Global Climate Observing System (GCOS) identified twenty-one fundamentally important Essential Climate Variables (ECVs) which are largely dependent on space-based EO [14]. ECVs are required for the validation of, and assimilation into, Earth system models to predict future climate change and thus the scarce resource of preferred Sun-synchronous orbits will become increasingly sought after for future EO missions. The use of low-thrust propulsion considered herein enables enhanced EO mission opportunities with currently available, or near-term, technology such as the QinetiQ T6 thruster, which will provide a thrust up to $230 \mathrm{mN}$ at a specific impulse above 4500 seconds for the BepiColombo mission [15].

Considering the application of extending the Sun-synchronous orbit it is noted that the ENVISAT orbit was selected for a variety of reasons, including instrument swath width for rapid global coverage and spacecraft 
Extension of the Sun-Synchronous Orbit author pre-print to Journal Guidance Control and Dynamics Copyright $\odot 2010$ by Malcolm Macdonald.

operations. Among the reasons for the ENVISAT orbit however is the desire to maintain continuous data collection from the profiling instruments, the Microwave Radiometer and the radar altimeter-2, which do not provide real global coverage but span a tight grid of measurements over the globe, first established by European Remote Sensing satellites 1 and 2 (ERS-1 and ERS-2). Such long-term, continuous data collection is critical for validation and assimilation into Earth system models to predict future climate change and as such, a change of orbit degrades the dataset. However, positioning a second spacecraft beside the nominal orbit, as demonstrated above, enhances the dataset by enabling the tight grid of measurements to be expanded. The application of the extended Sunsynchronous orbit is thus two-fold, enabling Sun-synchronous orbits at new orbit inclinations and enabling Sunsynchronous orbits on a given repeat ground-track at new orbit altitudes or inclinations to enhance the dataset collection of existing EO missions.

From Eq. 5 it is seen that the rate of change of the ascending node angle varies as the sinusoidal function of the argument of latitude. Therefore, assuming a circular orbit, the rate of change of the ascending node angle is zero at equatorial crossings regardless of the out-of-plane force applied. As such, any thrusting by the spacecraft within this region would be highly inefficient and spacecraft thrusting should be focused about the polar regions of the orbit. Note that such coast arcs require the thrust magnitude be increased such that the total delivered force about the orbit remains constant, however it can be shown that a coast arc of 25 degrees either side of the equator requires the force magnitude to be increased by less than $5 \%$. Furthermore, the thrust direction switching law described and used above could be replaced by a unidirectional thrust, for example, an attitude fixed solar sail, using an eccentric orbit with pericentre located over a planetary pole. On such an orbit, the rate of change of ascending node would be unbalanced about the semi-latus rectum/equatorial crossing, giving a secular variation in ascending node angle. Such a scenario is analogous to the Mercury Sun-Synchronous concept where a solar sail provides the required orbit perturbations for a Sun-Synchronous orbit at Mercury, otherwise not possible due to Mercury's high reciprocal of flattening [16].

\section{Conclusions}

It has been shown that the analytical derivation of the Sun-synchronous orbit can be extended to allow free selection of the orbit inclination and semi-major axis for a given repeat ground-track. The analytical derivation was considered using a numerical simulation, which showed that the extended and displaced Sun-synchronous orbit was valid. It was also shown that the required propellant to inject a spacecraft into such orbits was easily tolerable. The 
Extension of the Sun-Synchronous Orbit author pre-print to Journal Guidance Control and Dynamics Copyright $\odot 2010$ by Malcolm Macdonald.

level of thrust required to maintain either an extended Sun-synchronous orbit, or a displaced Sun-synchronous orbit was shown to be consistent with near-term technology under development for future planned missions including the BepiColombo mission.

\section{Acknowledgments}

This work was part-funded by ESA Contract Number 22349/09/F/MOS; Study on Gravity Gradient Compensation Using Low Thrust High Isp Motors.

\section{References}

${ }^{1}$ Roy, A.E., "Orbital Motion”, $4^{\text {th }}$ Edition, Taylor \& Francis, Abington, 2005, Chapter 7 \& 11. ISBN-13: 9780750310154 .

${ }^{2}$ Vallado, D.A., "Fundamentals of Astrodynamics and Applications", $3^{\text {rd }}$ Edition, Springer, New York, 2007, Chapter 11. ISBN-13: 978-0387718316.

${ }^{3}$ Duesmann, B., Barat, I., "ERS-2 and ENVISAT orbit control: current and future strategy - the impact on the interferometric baselines”, FRINGE 2007 Workshop, November 2007, ESA ESRIN in Frascati, Italy.

${ }^{4}$ Frerick, J., Duesmann, B., Canela, M., "2010 and Beyond - the ENVISAT Mission Extension”, Proc. 'Envisat Symposium 2007', Montreux, Switzerland, 23-27 April 2007, ESA SP-636, July 2007.

${ }^{5}$ M'Innes, C.R., "Solar Sailing: Technology, Dynamics and Mission Applications”, Springer-Praxis, Chichester, ISBN 1-85233-102-X, 1999, pp 120.

${ }^{6}$ Macdonald M., M'Innes C. R., “Analytical Control Laws for Planet-Centred Solar Sailing”, Journal of Guidance, Control, and Dynamics, Vol. 28, No. 5, 2005, pp. 1038-1048.

${ }^{7}$ McInnes, C.R., "Dynamics, Stability and Control of Displaced Non-Keplerian Orbits”, Journal of Guidance, Control and Dynamics, Vol. 21, No. 5, 1998, pp 799-805.

${ }^{8} \mathrm{M}^{\mathrm{c}}$ Kay, R., Macdonald, M., Bosquillon de Fresheville, F., Vasile, M., M'Innes, C., Biggs, J., "Non-Keplerian Orbits Using Low-thrust, High $I_{\mathrm{sp}}$ Propulsion Systems", IAC-09.C1.2.8, Electronic proceedings of the $60^{\text {th }}$ International Atsronautical Congress, Daejeon, Republic of Korea, October 2009.

${ }^{9}$ Dormand, J.R., Price, P.J., “A Family of Embedded Runge-Kutta Formulae”, Journal of Computing and Applied Mathematics, Vol. 6, 1980, pp $19-26$. 
Extension of the Sun-Synchronous Orbit author pre-print to Journal Guidance Control and Dynamics Copyright $\odot 2010$ by Malcolm Macdonald.

${ }^{10}$ Walker, M.J.H., Ireland, B., Owens, J., “A Set of Modified Equinoctial Elements”, Celestial Mechanics, Vol. 36, 1985, pp. 409-419.

${ }^{11}$ Betts, J. T., “Optimal Interplanetary Orbit Transfers by Direct Transcription”, Journal of Astronautical Sciences, Vol. 42, No. 3, July-Sept. 1994, pp 247-268.

${ }^{12}$ Vasile, M., and Bernelli-Zazzera, F., "Targeting a Heliocentric Orbit Combining Low-Thrust Propulsion and Gravity Assist Manoeuvres," Operations Research in Space and Air, Applied Optimization, Vol. 79, Kluwer Academic, Norwell, MA, 2003.

13 Vasile, M., and Bernelli-Zazzera, F., "Optimizing Low-Thrust and Gravity Assist Manoeuvres to Design Interplanetary Trajectories,” Journal of the Astronautical Sciences, Vol. 51, No. 1, Jan.-Mar. 2003, pp. 13-35.

${ }^{14}$ European Space Agency - Earth Observation Programme Board. "Global Monitoring of Essential Climate Variables", ESA/PB-EO(2009)32, February 2009.

${ }^{15}$ Wallace, N., "Testing of the QinetiQ T6 Thruster in Support of the ESA BepiColombo Mercury Mission", Proceedings of the $4^{\text {th }}$ International Spacecraft Propulsion Conference (ESA SP-555), Chia Laguna (Cagliari), Sardinia, Italy. Ed. A.Wilson. Published on CDROM., 2-9 June, 2004. Available from: http://articles.adsabs.harvard.edu/full/2004ESASP.555E..70W, cited 24 August 2009.

${ }^{16}$ Leipold, M. E., andWagner, O., "Mercury Sun-SynchronousPolar Orbits Using Solar Sail Propulsion,” Journal of Guidance, Control, and Dynamics, Vol. 19, No. 6, 1996, pp. 1337-1341. 